\title{
A Case of Ectopic Peritoneal Paragonimiasis Mimicking Diverticulitis or Abdominal Abscess
}

\author{
Min Jae Kim¹, Sung-Han Kim¹, Sang-Oh Lee', Sang-Ho Choi', Yang Soo Kim¹, Jun Hee Woo', Yong Sik Yoon², \\ Kyung Won $\mathrm{Kim}^{3}$, Jaeeun $\mathrm{Cho}^{4}$, Jong-Yil Chai, ${ }^{4,5}$, Yong Pil Chong ${ }^{1, *}$

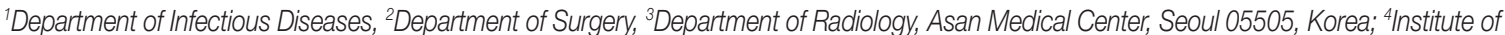 \\ Parasitic Diseases, Korea Association of Health Promotion, Seoul 07049, Korea; ${ }^{5}$ Department of Parasitology and Tropical Medicine, Seoul National \\ University College of Medicine, Seoul 03080, Korea
}

\begin{abstract}
Paragonimiasis is a parasitic disease caused by Paragnonimus species. The primary site of infection is the lung, and extrapulmonary involvement is also reported. When infected with Paragonimus westermani, which is the dominant species in Korea, the central nervous system is frequently involved along with the liver, intestine, peritoneal cavity, retroperitoneum, and abdominal wall. Ectopic paragonimiasis raises diagnostic challenge since it is uncommon and may be confused with malignancy or other inflammatory diseases. Here, we report an ectopic paragonimiasis case initially presented with recurrent abdominal pain. The patient developed abdominal pain 3 times for the previous 3 years and the computed tomography (CT) of the abdomen revealed fluid collection with wall enhancement. Recurrent diverticulitis was initially suspected and part of the ascending colon was resected. However, the specimen showed intact colon wall without evidence of diverticulitis and multiple parasite eggs and granulomas were found instead. The size of about $70 \mu \mathrm{m}$, the presence of an operculum and relatively thick egg shell suggested eggs of Paragonimus species. With appropriate exposure history and a positive antibody test, the definitive diagnosis was made as peritoneal paragonimiasis.
\end{abstract}

Key words: Paragonimus westermani, paragonimiasis, peritoneaum, abscess, parasite egg

\section{INTRODUCTION}

Paragonimiasis is a parasitic disease caused by Paragonimus species or lung flukes. Most common site of infection is the lung, and the infected person usually presents with cough, hemoptysis, and pleural effusion. However, extrapulmonary involvement is also reported. In cases infected with Paragonimus westermani, which is the dominant species in Korea, the central nervous system involvement comprised $45 \%$ of the total extrapulmonary paragonimiasis [1]. We present here a case of peritoneal paragonimiasis, which was initially considered as recurrent diverticulitis and intra-abdominal abscess.

\section{CASE RECORD}

A 45-year-old male was admitted to our hospital for recur-

• Received 13 February 2017, revised 25 May 2017, accepted 1 Jun 2017.

*Corresponding author (drchong@amc.seoul.kr)

(c) 2017. Korean Society for Parasitology and Tropical Medicine

This is an Open Access article distributed under the terms of the Creative Commons Attribution Non-Commercial License (http://creativecommons.org/licenses/by-nc/4.0) which permits unrestricted non-commercial use, distribution, and reproduction in any

medium, provided the original work is properly cited. rent abdominal pain. The abdominal pain first developed suddenly 3 years before this admission. The patient remembered that it was localized in the right lower quadrant area, and its intensity was about 4 to 5 on a scale of 0 to 10 , with 10 indicating the most severe pain. He denied febrile sense or chills. On the next day, computed tomography (CT) of the abdomen and pelvis was performed and revealed fluid dispersion and fat infiltration around the cecum and small bowel mesentery. Although there was no colonic wall thickening, a presumptive diagnosis of acute diverticulitis was made. The abdominal pain was improved 4 days after intravenous antibiotics treatment and fasting. Later, he underwent colonoscopy and esophagogastroduodenoscopy, and no abnormal finding was found.

At 27 months after the initial episode (10 months before this admission), the abdominal pain developed again at the same area. The patient also denied any systemic symptoms. The CT of the abdomen and pelvis was repeated and revealed several thick-walled cavitary lesions, which are suggestive of intra-abdominal abscesses with adjacent peritoneal fat infiltration. Recurred diverticulitis and peritoneal abscess was suspected, and surgical resection of the cecum was discussed with 

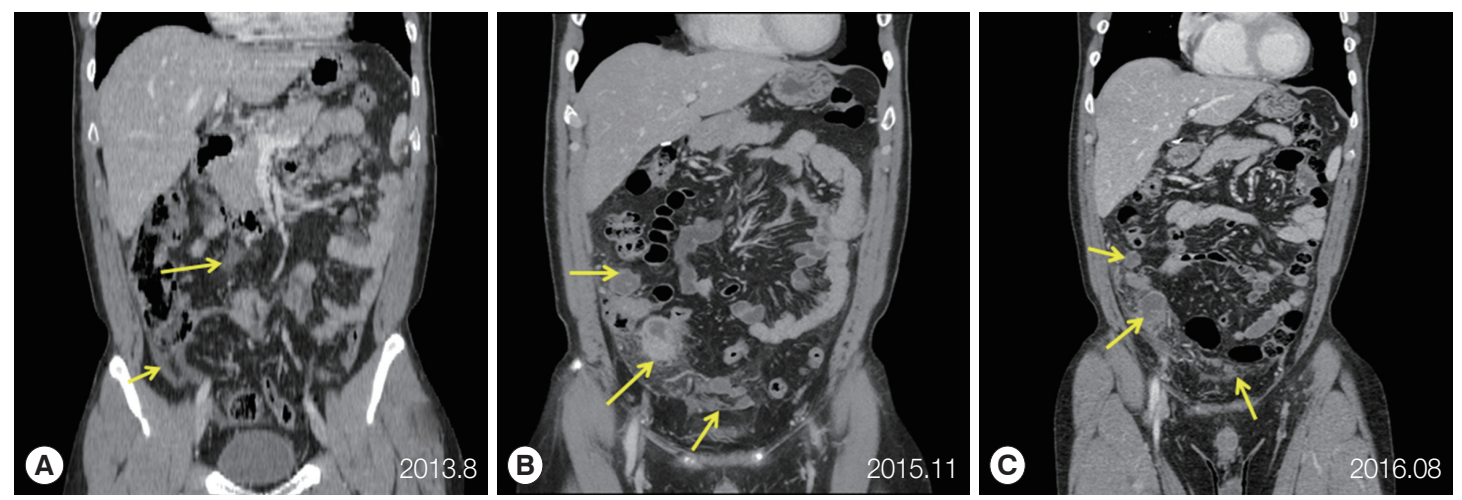

Fig. 1. Serial CT findings of peritoneal paragonimiasis. (A) A coronal CT scan performed on first episode, 2013, showing fluid dispersion and fat infiltration around the cecum and small bowel mesentery (arrows). (B) CT images performed on second episode, 2015, demonstrating several thick-walled cavitary lesions (arrows) suggestive of intra-abdominal abscess with adjacent peritoneal fat infiltration. (C) A CT performed on recent episode showing that the overall extent of intra-abdominal abscesses (arrows) and peritoneal fat infiltration was slightly decreased, but the majority of lesions remained.

the patient. Since he refused the surgery, medical treatment with intravenous antibiotics started and resulted in the improvement of symptoms in 1 week.

At 2 weeks before admission, the patient reported abdominal pain for the third time. The pain started over the whole abdomen, and later it was localized at the right lower quadrant area. The patient complained of continuous pain and rated its intensity as 6 to 7 on a scale of 0 to 10 . This time he felt febrile sense and chills. Other gastrointestinal symptoms, such as anorexia, nausea, vomiting, and diarrhea were absent. On examination, localized tenderness and rebound tenderness was found at the right lower quadrant area. A complete blood count showed white-cell count $5,800 \times 10^{3}$ per $\mathrm{mm}^{3}$ with $56.7 \%$ of neutrophils and $3.3 \%$ of eosinophils. Total bilirubin was slightly increased as $1.5 \mathrm{mg} / \mathrm{dl}$, and the results of other liver function tests, such as aspartate aminotransferase, alanine aminotransferase, alkaline phosphatase, and glutamyl transferase were within normal range. C-reactive protein was elevated to $4.66 \mathrm{mg} / \mathrm{dl}$. The chest radiography showed no active lung lesions. The repeated CT of the abdomen and pelvis showed that the majority of the previous peritoneal lesions remained although the overall extent of intra-abdominal abscesses and peritoneal fat infiltration was slightly decreased. Colonic wall thickening or enhancement was not definite (Fig. 1).

Intravenous antibiotics infusion started with piperacillin and tazobactam. In consideration of his recurrent episodes, the patient underwent laparoscopic right hemicolectomy after 2 weeks of antibiotics treatment. Multiple abscess-like lesions were found adjacent to the proximal colon and also numerous peritoneal nodules were identified. The terminal ileum, ce- cum, and proximal ascending colon were removed along with adjacent lesions. Multiple biopsies also were performed on peritoneal nodules. When the specimen was examined, colonic wall revealed no abnormality. The appendix showed reactive follicles and acute serositis, and lymph nodes showed reactive hyperplasia. The abscess was shown as necrotizing granulomas with eosinophilic infiltrations and many parasite eggs. Peritoneal nodules were identified as a granuloma, and parasite eggs were identified inside.

When questioned about the consumption of raw freshwater fish or crab, the patient disclosed that he frequently ate the soybean sauce marinated crabs and boiled snails. He remembered that he visited Thailand, the Philippines, China, and Turkey during the previous 5 years. However, he could not recall any consumption of raw food and freshwater exposure abroad except swimming in swimming pools in hotels. Antibodies for Clonorchis sinensis, P. westermani, Schistosoma haematobium, cysteicercus, and Anisakis species were checked with ELISA method. Only antibodies for P. westermani were positive. When the surgical specimen was carefully examined, the size of the eggs was about 60 to $70 \mu \mathrm{m}$ in the longest diameter. The thick egg shell and the operculum suggested that the eggs were those of Paragonimus species (Fig. 2). Collectively, the diagnosis of peritoneal paragonimiasis was made, and the patient was treated with praziquantel $25 \mathrm{mg} / \mathrm{kg} 3$ times a day for 2 days.

\section{DISCUSSION}

Here, we presented a case of peritoneal paragonimiasis who suffered from recurrent abdominal pain. Since the thick- 

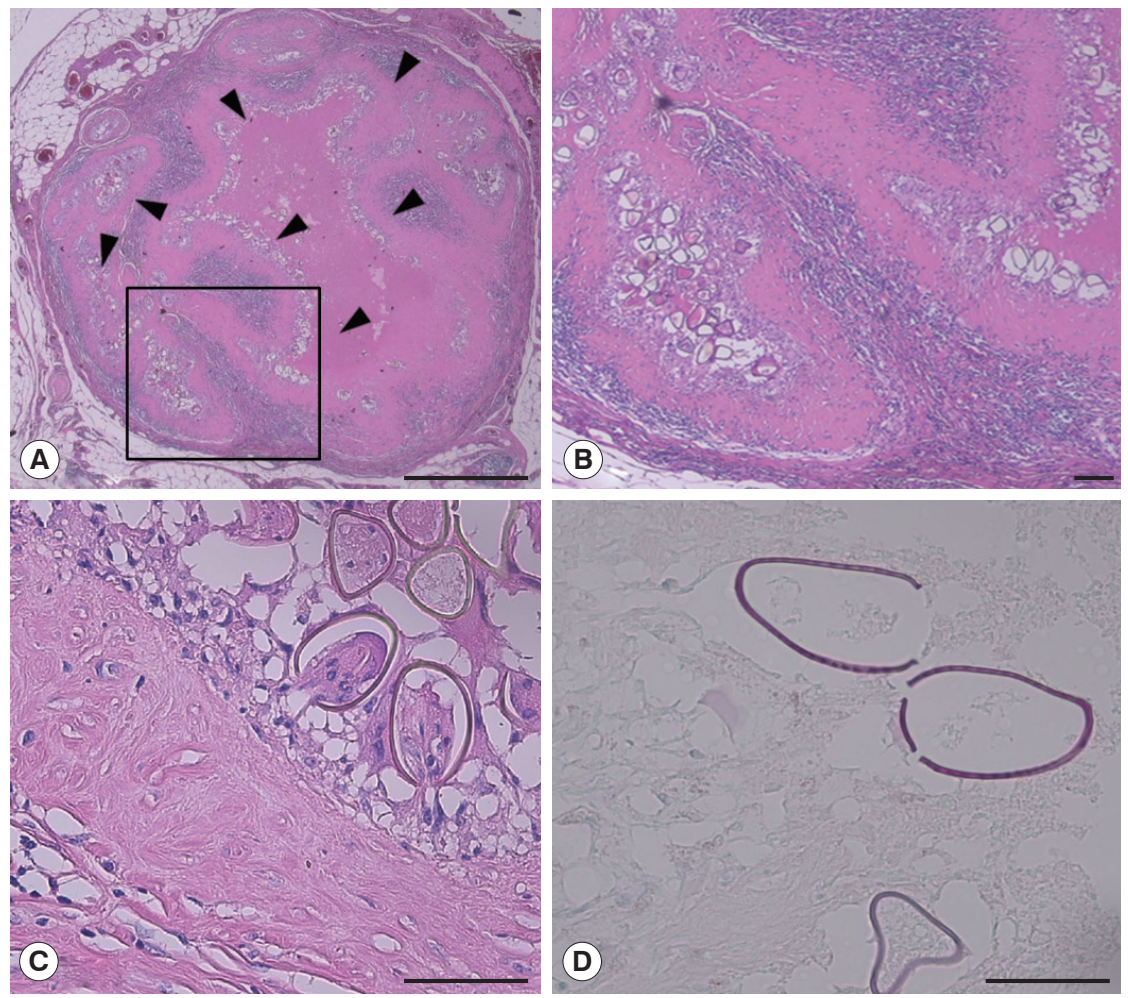

Fig. 2. Pathologic findings of the peritoneal nodule. (A) Granuloma containing numerous parasite eggs (arrow head). A part of the nodule is shown enlarged in (B). The characteristics of the parasite eggs are shown in (C) and (D). Bar=1 mm in (A), $100 \mu \mathrm{m}$ in (B) and $50 \mu \mathrm{m}$ in (C) and (D).

walled cavitary lesions were adjacent to the cecum on abdominal CT, recurrent diverticulitis and intra-abdominal abscess were initially considered. Only after the surgery, paragonimiasis was diagnosed based on the pathologic findings and subsequent serology.

When the abdominal pain is localized to the right lower quadrant, usual differential diagnosis includes acute appendicitis, diverticulitis, colitis, and inflammatory bowel diseases. Some of these diseases can cause recurrent episodes. For example, underlying colonic diverticulosis could result in repeated diverticulitis and unresolved vascular problem can cause recurrent ischemic colitis. In our case, the initially identified lesions on CT were peritoneal fluid dispersion with fat infiltration. They developed into thick-walled cavitary lesions containing dirty fluid or debris over 27 months and remained in the similar position. The chronic nature of the lesions and an absence of diverticulosis in colonoscopy suggested some kinds of chronic peritoneal inflammatory diseases, including parasite infections. The definitive diagnosis was made as peritoneal paragonimiasis after a surgery.

Paragonimiasis is a parasitic disease caused by Paragonimus species. Humans are usually infected when they consume inadequately cooked crabs or crayfish which contain the encysted metacercariae of Paragonimus species. In Korea, P. westermani is the most common etiology, and soybean sauce marinated freshwater crabs are well-known as the source of human paragonimiasis. When a metacercaria contained in the crab is swallowed, it excysts in the small intestine, penetrates the intestinal wall to the peritoneal cavity, and moves through the diaphragm and pleura into the lung. The larva makes a cystlike capsule in the lung and matures inside the cavity. Clinical symptoms of pulmonary paragonimiasis occur when the cyst capsule ruptures into the bronchioles. The parasite eggs are discharged through the airway when the infected patients cough.

Although the lung is the most common site of infection, other organs such as the central nervous system, liver, intestine, peritoneal cavity, retroperitoneum, and abdominal wall are also known to be involved [2-7]. In many cases, mass-like lesions or abscess were found in the involved organs. Malignancy is a concern, so surgical resection is usually performed to treat the abscess and to exclude the malignancy. On surgical 
removal, worms or eggs of Paragonimus species could be found. When "PubMed" and "KoreaMed" were searched with terms of "ectopic paragonimiasis", 10 articles of either English or Korean language were found after the year 2010 [6,8-16]. These cases included 5 cases of subcutaneous infection, 5 of intra-abdominal infection, 2 of central nervous system infection, and 1 case of mediastinal cavity infection. All the cases underwent either radiological tissue biopsy or surgery, and the final diagnoses were made from pathological examinations revealing the parasite or eggs.

The definitive diagnosis of ectopic paragonimiasis can be made when worms or eggs of characteristic shape are found on a surgical specimen. When the specimen could not reveal the typical characteristics of Paragonimus worm or eggs, molecular approaches, such as PCR can be helpful [17]. Some cases are diagnosed with exposure history, radiological findings, and immunological test. Commonly, ELISA is used to measure antibodies to Paragonimus species. However, a positive ELISA test does not always imply that active infection is present, since it took 4 to 18 months for the antibody level to decrease to a normal level [18]. Praziquantel $25 \mathrm{mg} / \mathrm{kg} 3$ times for 2-3 days are usually used to treat ectopic paragonimiasis, including cerebral paragonimiasis [19]. Surgery is usually performed when the lesions are relatively superficial and easy to remove or when the dead worm accompanied complications [17].

In addition to Paragonimus species, the metacercariae of Fasciola hepatica migrate to the peritoneal cavity to reach the liver and bile duct, the usual site of infection. Occasionally, the metacercaria is entrapped in the peritoneum, grows to be an adult, and produces abscess [20,21]. Larvae of Anisakis species and the plerocercoid of Spirometra species (=sparganum) can also penetrate the wall of the gastrointestinal tract and could be found in the peritoneal cavity $[22,23]$. These parasitic infections are known to produce varying degrees of mesenteric infiltrations, peritoneal nodules, and peritoneal thickening in CT of the abdomen [24]. In our case, the underlying problem for the multiple peritoneal abscesses was considered to be diverticulitis, but later found to be the granulomatous inflammation caused by parasite eggs.

The exact prevalence of human paragonimiasis in a defined community is hard to estimate since no single satisfactory diagnostic method exists. Regular national intestinal parasite surveys examine the stools of the systematically sampled population. Since the passage of parasite eggs in the stool only occurs in a limited number of patients, the eggs of Paragonimus species are hardly detected even in national surveys since 1981 . Only several seroprevalence data suggests that the prevalence of paragonimiasis is decreasing [25]. Our case clearly shows that the human paragonimiasis is still endemic in Korea, and ectopic paragonimiasis should be included in the differential diagnosis of peritoneal inflammatory lesions.

\section{CONFLICT OF INTEREST}

The authors declare that they have no conflict of interest related to this study.

\section{REFERENCES}

1. Chai JY. Paragonimiasis. Handb Clin Neurol 2013; 114: 283296.

2. Kim EA, Juhng SK, Kim HW, Kim GD, Lee YW, Cho HJ, Won JJ. Imaging findings of hepatic paragonimiasis: a case report. J Korean Med Sci 2004; 19: 759-762.

3. Liu CT, Chen YC, Chen TH, Barghouth U, Fan CK. Intestinal paragonimiasis with colonic ulcer and hematochezia in an elderly Taiwanese woman. Korean J Parasitol 2012; 50: 349-352.

4. Jeong WK, Kim Y, Kim YS, Park DW, Park CK, Baek HK, Park YW. Heterotopic paragonimiasis in the omentum. J Comput Assist Tomogr 2002; 26: 1019-1021.

5. Kim JY, Kang CM, Choi GH, Yang WI, Sim SB, Kwon JE, Kim KS, Choi JS, Lee WJ, Kim BR. Laparoscopic excision of intra-abdominal paragonimiasis. Surg Laparosc Endosc Percutan Tech 2007; 17: 556-558.

6. Lee $\mathrm{CH}$, Kim JH, Moon WS, Lee MR. Paragonimiasis in the abdominal cavity and subcutaneous tissue: report of 3 cases. Korean J Parasitol 2012; 50: 345-347.

7. Lee YH, Park EH, Kim WC, Choi YD, Park JH. A case of pelvic paragonimiasis combined with myoma uteri and pelvic inflammatory disease. Korean J Parasitol 1993; 31: 295-297.

8. Han SY, Lee WH, Yu JS, Lee SR, An JY, Koh JH, Park Y. A case of pleuropulmonary paragonimiasis with a breast abscess as the ectopic site. Korean J Med 2011; 81: 502-507.

9. Kim AY. Heterotopic paragonimiasis presented by intra-abdominal masses. Korean J Gastroenterol 2013; 61: 351-353 (in Korean).

10. Kim JS, Seo BC, Kim YJ, Jun YJ. Paragonimiasis in the abdominal subcutaneous tissue: a case report. J Korean Soc Plast Reconstr Surg 2010; 37: 485-488 (in Korean).

11. Kim SH, Kim HJ, Lee JI, Kye BH, Oh SN, Jung CK, Kang WK, Kim JG, Oh ST. A case of intra-abdominal heterotopic paragonimiasis combined with rectal cancer. J Korean Soc Coloproctol 2010; 26: 157-160 (in Korean).

12. Koh EJ, Kim SK, Wang KC, Chai JY, Chong S, Park SH, Cheon JE, Phi JH. The return of an old worm: cerebral paragonimiasis pre- 
senting with intracerebral hemorrhage. J Korean Med Sci 2012; 27: $1428-1432$

13. Lim SH, Choi H, Lee KY, Lee YJ, Koh SH. Cerebral paragonimiasis presenting as recurrent hemorrhagic stroke without pulmonary symptoms. J Korean Neurol Assoc 2011; 29: 371-373.

14. Oh CR, Kim MJ, Lee KH. A case of intra-abdominal paragonimiasis mimicking metastasis of lung cancer diagnosed by endoscopic ultrasound-guided fine needle aspiration. Korean J Gastroenterol 2015; 66: 41-45 (in Korean).

15. Park SA, Lee SH, Ko SH, Kim JG, Park SY, Yoo JY, Nam HW, Ahn YB. A case of incidentally diagnosed adrenal paragonimiasis. Endocrinol Metab 2011; 26: 97-100.

16. Ra YJ, Ahn HY, Kim YS, Choi KU. Isolated and ectopic mediastinal paragonimiasis without any pulmonary or pleural lesion. Korean J Thorac Cardiovasc Surg 2010; 43: 553-556 (in Korean).

17. Blair D. Paragonimiasis. Adv Exp Med Biol 2014; 766: 115-152.

18. Cho SY, Kim SI, Kang SY, Kong Y, Han SK, Shim YS, Han YC. Antibody changes in paragonimiasis patients after praziquantel treatment as observed by ELISA and immunoblot. Korean J Parasitol 1989; 27: 15-21.

19. Chen J, Chen Z, Lin J, Zhu G, Meng H, Cui G, Wu N, Hu R, Pan
J, Zou Y, Feng H. Cerebral paragonimiasis: a retrospective analysis of 89 cases. Clin Neurol Neurosurg 2013; 115: 546-551.

20. Song KD, Lim JH, Kim MJ, Jang YJ, Kim JW, Cho SH, Kwon JH. Peritoneal manifestations of fascioliasis on CT images: a new observation. Abdom Imaging 2013; 38: 839-843.

21. Kim AJ, Choi CH, Choi SK, Shin YW, Park YK, Kim L, Choi SJ, Han JY, Kim JM, Chu YC, Park IS. Ectopic human Fasciola hepatica infection by an adult worm in the mesocolon. Korean J Parasitol 2015; 53: 725-730.

22. Cespedes M, Saez A, Rodriguez I, Pinto JM, Rodriguez R. Chronic anisakiasis presenting as a mesenteric mass. Abdom Imaging 2000; 25: 548-550.

23. Lee JH, Yu JS, Park MS, Lee SI, Yang SW. Abdominal sparganosis presenting as an abscess with fistulous communication to the bowel. AJR Am J Roentgenol 2005; 185: 1084-1085.

24. Kim SY, Ha HK. Peritoneal manifestations of parasitic infection. Abdom Imaging 2008; 33: 172-176.

25. Lee MK, Hong SJ, Kim HR. Seroprevalence of tissue invading parasitic infections diagnosed by ELISA in Korea. J Korean Med Sci 2010; 25: 1272-1276. 